\title{
A deconvolution technique to correct deep images of galaxies from instrumental scattered light ${ }^{\star}$
}

\author{
E. Karabal ${ }^{1,2}$, P.-A. Duc ${ }^{1}$, H. Kuntschner ${ }^{2}$, P. Chanial ${ }^{3}$, J.-C. Cuillandre ${ }^{1,4}$, and S. Gwyn ${ }^{5}$ \\ ${ }^{1}$ Laboratoire AIM Paris-Saclay, CEA/Irfu/SAp, CNRS, Université Paris Diderot, 91191 Gif-sur-Yvette Cedex, France \\ e-mail: emin.karabal@cea.fr \\ 2 European Southern Observatory, Karl-Schwarzschild-Str. 2, 85748 Garching, Germany \\ 3 Astroparticule et Cosmologie (APC), CNRS-UMR 7164, Université Paris 7 Denis Diderot, 10 rue Alice Domon et Léonie Duquet, \\ 75205 Paris Cedex 13, France \\ ${ }^{4}$ Observatoire de Paris, PSL Research University, France \\ 5 NRC, National Research Council of Canada, 5071 West Saanich Rd, Victoria, British Columbia, V9E 2E7, Canada
}

Received 28 October 2016/ Accepted 14 December 2016

\begin{abstract}
Deep imaging of the diffuse light that is emitted by stellar fine structures and outer halos around galaxies is often now used to probe their past mass assembly. Because the extended halos survive longer than the relatively fragile tidal features, they trace more ancient mergers. We use images that reach surface brightness limits as low as 28.5-29 mag $\operatorname{arcsec}^{-2}$ ( $g$-band) to obtain light and color profiles up to 5-10 effective radii of a sample of nearby early-type galaxies. These were acquired with MegaCam as part of the CFHT MATLAS large programme. These profiles may be compared to those produced using simulations of galaxy formation and evolution, once corrected for instrumental effects. Indeed they can be heavily contaminated by the scattered light caused by internal reflections within the instrument. In particular, the nucleus of galaxies generates artificial flux in the outer halo, which has to be precisely subtracted. We present a deconvolution technique to remove the artificial halos that makes use of very large kernels. The technique, which is based on PyOperators, is more time efficient than the model-convolution methods that are also used for that purpose. This is especially the case for galaxies with complex structures that are hard to model. Having a good knowledge of the point spread function (PSF), including its outer wings, is critical for the method. A database of MegaCam PSF models corresponding to different seeing conditions and bands was generated directly from the deep images. We show that the difference in the PSFs in different bands causes artificial changes in the color profiles, in particular a reddening of the outskirts of galaxies having a bright nucleus. The method is validated with a set of simulated images and applied to three representative test cases: NGC 3599, NGC 3489, and NGC 4274, which exhibits a prominent ghost halo for two of them. This method successfully removes this.
\end{abstract}

Key words. galaxies: evolution - galaxies: elliptical and lenticular, $\mathrm{cD}$ - galaxies: stellar content - galaxies: photometry techniques: photometric - techniques: image processing

\section{Introduction}

The mass growth and morphological evolution of galaxies is described by different scenarios in the literature: gas accretion from cosmological filaments, internal secular evolution and series of galaxy mergers of different mass ratios and gas fractions. Depending on the dominant scenario, the simulations modeling these processes predict specific observables for the resulting galaxy, such as stellar age and metallicity profiles probed by spectroscopy, as well as light and color profiles traced by photometry (e.g. Naab et al. 2007; Bullock \& Johnston 2005; Oser et al. 2010; Cooper et al. 2010; Pillepich et al. 2015).

While the inner regions of galaxies have been studied thoroughly, the extended parts are still an immature but rapidly growing field of research. Multiple programs are being carried out to explore these regions that hold the imprints of recent and more ancient mergers. They make use of either stellar counts for local galaxies, or the diffuse light revealed by deep imaging for

* The library of PSFs (FITS files) is only available at the CDS via anonymous ftp to cdsarc.u-strasbg. fr $(130.79 .128 .5)$ or via http://cdsarc.u-strasbg.fr/viz-bin/qcat?]/A+A/601/A86 more distant ones (Mihos et al. 2005; Martínez-Delgado et al. 2010; Janowiecki et al. 2010; Abraham \& van Dokkum 2014; Duc et al. 2015; Iodice et al. 2016). New imaging techniques have been developed (Mihos et al. 2005; Ferrarese et al. 2012; van Dokkum et al. 2014), which enable us to reach the very low surface brightness levels needed to investigate the extended stellar structures of galaxies. Deep images reveal the fine structures produced by recent mergers (shells, streams, tails), as well as the outer stellar halos, which cumulate material accreted during more ancient collisions. Stellar halos in extended regions survive longer than the fine structures. Unfortunately their photometric analysis suffers from various sources of contamination.

The challenges of deep imaging require elaborate techniques to reach the desired sensitivity in the extended parts of light and color profiles. Various contamination sources hamper the integrated light analysis of the halos, in particular galactic cirrus and instrumental contamination. Owing to its complex filamentary structure (Miville-Deschenes et al. 2016), and nonmonochromatic colours, the optical emission from Galactic cirrus cannot be easily subtracted; heavily cirrus polluted regions should thus be avoided. Instrumental contamination affects all 
fields; some can be addressed by advanced flat-fielding procedures and sky subtraction; others, like reflections within the camera, which produce artificial halos and ghosts, are much more complex to model and remove (Slater et al. 2009; Duc et al. 2015). The importance of correcting for light scattering is well documented (Michard 2002; Slater et al. 2009; Sandin 2015; Trujillo \& Fliri 2016) but the correction process is challenging. In particular, it requires the availability of point spread functions (PSFs) measured up to large radius at the location of the target on the detector. Minimising artificial halos should, in principle, be done at the instrumental level. The scattering in classical cameras may be reduced with an anti-reflective coating on the detector and on the optical elements (Mihos et al. 2005) or largely eliminated by alternative imaging systems, such as photo-lens array (Abraham \& van Dokkum 2014).

Sandin $(2014,2015)$ investigate the impact of ghost halos on galaxy profiles by convolving galaxy models with PSFs that correspond to a variety of instruments. He quantifies the importance of scattering as a function of radius for various morphological types. Duc et al. (2015) note the wavelength dependence of the scattering effect which, for the MegaCam instrument, climax in the $r$ band. The variation of the PSF shape from one band to another creates artificial reddening in the outer color profiles of galaxies. Trujillo \& Fliri (2016) apply a model convolution technique to estimate and remove light scattering: a pre-determined intrinsic galaxy model is convolved with the PSF, and the best solution is iteratively found by fitting the convolved model to the data. With this method, having a multi-component model that minimises residuals in the model-subtracted image is fundamental: positive or negative residuals cause under-estimation or over-estimation of the scattered light. Building a specific multi-component galaxy model interactively is time consuming and becomes prohibitive for large surveys of massive, complex, galaxies.

In this paper, instead of using the model-convolution technique, we present our efforts to directly deconvolve images and remove artificial halos of galaxies. The deconvolution method relies on large kernels and uses PyOperators ${ }^{1}$ (Chanial \& Barbey 2012), which are time-efficient python tools. Our analysis is based on CFHT/MegaCam deep images that were acquired as part of the MATLAS (Mass Assembly of early-Type GaLAxies with their fine Structures) large programme (Duc et al. 2015), as well as simulated images used to validate the method.

This paper is structured as follows: in Sect. 2, we present the MATLAS images and characterize their ghost halos; we describe the method to determine the point spread functions to large radii, detail the deconvolution technique and the routines to derive light and color profiles. In Sect. 3, we present the results obtained with images of simulated galaxies and apply the method for images of three real galaxies - NGC 3489, NGC 3599, and NGC 4274 - all of which exhibit a variety of artificial halos. In Sect. 4, we discuss the limitations of the method owing to uncertainties on the PSF and potential issues dealing with saturated images. In Sect. 5, we draw conclusions.

\section{Data and method}

\subsection{The ghosts on MATLAS images}

MATLAS is a deep imaging survey that uses MegaCam on the CFHT (Boulade et al. 2003) to acquire images of nearby early-type galaxies from the sample of the ATLAS ${ }^{3 \mathrm{D}}$ project

\footnotetext{
1 http://pchanial.github.io/pyoperators/
}

(Cappellari et al. 2011). The ATLAS ${ }^{3 \mathrm{D}}$ sample is volumelimited (within $42 \mathrm{Mpc}$ ) and includes galaxies with dynamical mass in the range $\sim 10^{9.6}-10^{11.8} M_{\odot}$ (Cappellari et al. 2013).

MATLAS images are obtained by stacking 6-7 individual images, each of which has a 6-min exposure time. The individual pointings are made with large offsets from one another (in the range of 2-14 arcmin) to enable the detection of extended lowsurface-brightness features. The images are collected in the $g^{\prime}$ and $r^{\prime}$-band and, additionally, some galaxies are observed in $u^{*}$ and $i^{\prime 2}$. In most cases, the target galaxies are located close to the center of the $1 \times 1$ square degree stacked image. Galaxies that are within the field of an already observed object were not reobserved; some are thus located closer to the edge at locations where the shape of the PSF is significantly changed with respect to that measured in the central regions. Further details on the observing strategy and data reduction technique can be found in Duc et al. (2015).

The MATLAS images were usually obtained under good seeing conditions and benefit from the excellent image quality of the MegaCam camera, while reaching low local surface brightness limits (28.5-29 mag $\operatorname{arcsec}^{-2}$ in the $g$-band). They are, however, subject to prominent scattering effect owing to the complex optical structure of the instrument. Multiple internal reflections create characteristic extended interleaved halos (from now on referred to as ghosts) around bright objects, stars, as well as galaxies.

The ghosts are due to the light coming to the camera, which is being reflected back and forth between the CCD and the other optical elements, such as the lenses and filter wheels. The origin and the physical processes behind these ghosts were investigated by Slater et al. (2009). The presence of ghosts alters the photometric analysis, i.e. changing the light and color profiles, especially in the outskirts of galaxies, beyond the $\gtrsim 24$ mag $\operatorname{arcsec}^{-2}$ isophote. The radius of the most prominent ghost seen in the MegaCam deep images is $\sim 3.5$ arcmin.

As illustrated in Fig. 1, ghosts are present everywhere in the images, contributing to the image background; however, they are directly visible only around bright objects. The two sources of ghosts - bright stars and galactic nuclei - cause external and self-contamination, respectively. Different methods are applied to remove them; the ghosts of bright stars can be easily identified in the images, manually modeled, and subtracted. On the other hand, reflection halos surrounding galaxies are more complex, since the light that is contributed to them is extended. Besides, these ghosts cannot be easily identified and separated from the stellar halo of the galaxy, especially when the latter is extended. Therefore, only a deconvolution technique can properly remove them. We note that this process does not eliminate the ghosts around bright stars since they are saturated.

\subsection{Subtraction of the ghosts around the bright stars close to the galaxies}

The extended halos around the bright stars, which are close to the target galaxies, contaminate their stellar halos, and need to be subtracted.

On individual images, these ghosts consist of multiple annuli that partially overlap each other which, depending on their position on the CCDs, and bands used, have different angular sizes, center, and brightnesses. The stacking procedure of these individual images that have large mutual spatial offsets (see Fig. 2) adds to their complexity. A manual interactive procedure was

\footnotetext{
2 Noted hereafter, $u, g, r$ and $i$.
} 


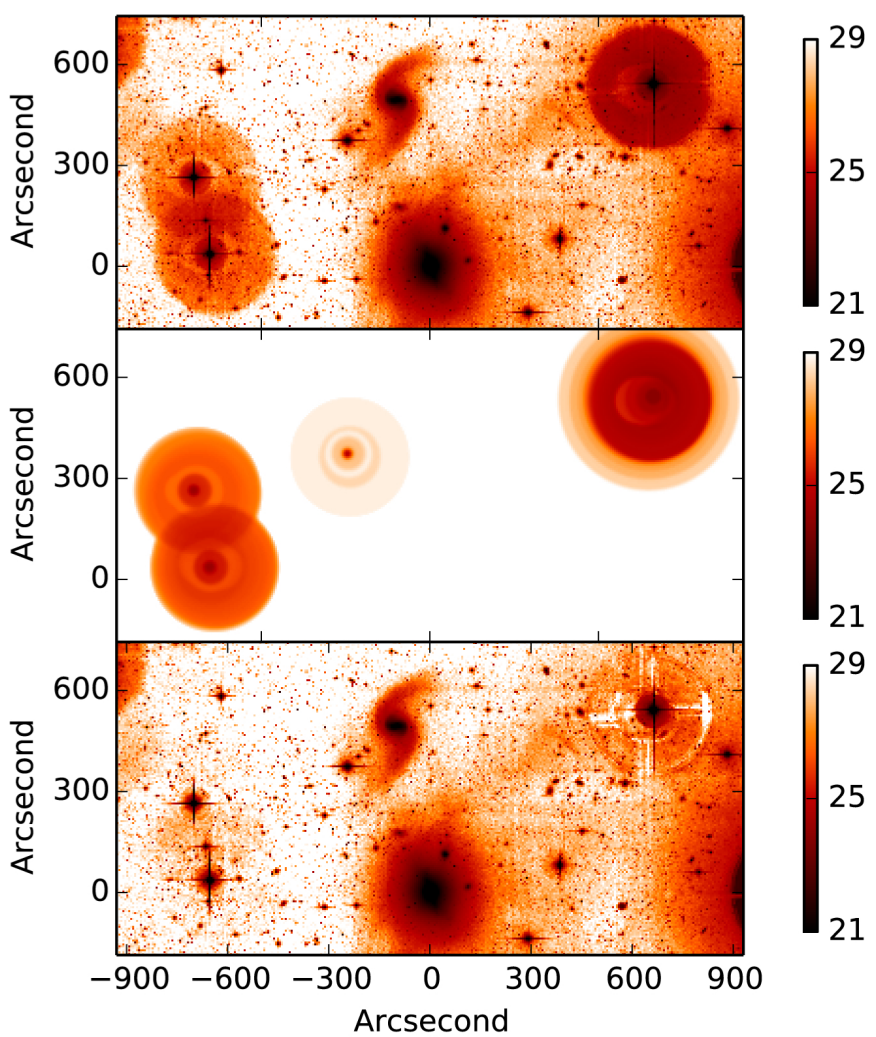

Fig. 1. Surface brightness maps in $r$-band of the stellar ghosts around NGC 3414 (top), the ghost models (middle) and the ghost subtracted image (bottom). The color bar to the right represents the surface brightness level. The target galaxy is centered at the $[0,0]$ coordinate of the map. The maps are blurred with a Gaussian $\left(\right.$ sigma $\left.=0.55^{\prime \prime}\right)$ to make faint regions visible.

then set up to generate models of the stacked halos: series of annuli with manually adjusted values were constructed, starting with the external regions. Each annulus is subtracted from the image and the residual image is iteratively processed until the halo disappears and a flat background is reached. The procedure is illustrated in Fig. 3. We note that the method is unable to model properly gaps with lower intensity, which are present between some annuli especially in the individual images. On the stacked images, these are, however, partly filled (see Fig. 3) and modeling them would anyway be too complex. The light-fitting procedure starts at a radius of $200^{\prime \prime}$, and is stopped at $\sim 50^{\prime \prime}$. Modeling the profiles further in would not help a great deal for the purpose of decontaminating the galaxy halo by the stellar ghost.

This process creates sharp edges while the boundary of the original ghosts were smoother, owing to the stacking procedure on images obtained with large offsets. To take this into account, a Gaussian blur was applied on the final ghost model. The shape of the halos is wavelength-dependent, as further discussed in Sect. 2.3. Thus different models had to be constructed for each band. Finally, the ghost halo models were subtracted from the images, as illustrated in Fig. 1 for the field of galaxy NGC 3414.

\subsection{Determination of the point spread function}

Deconvolution techniques such as the one presented here in principle require a perfect knowledge of the PSF at the location, time, and wavelength of the observations, and to determine it from the innermost to the outermost regions. In the optical

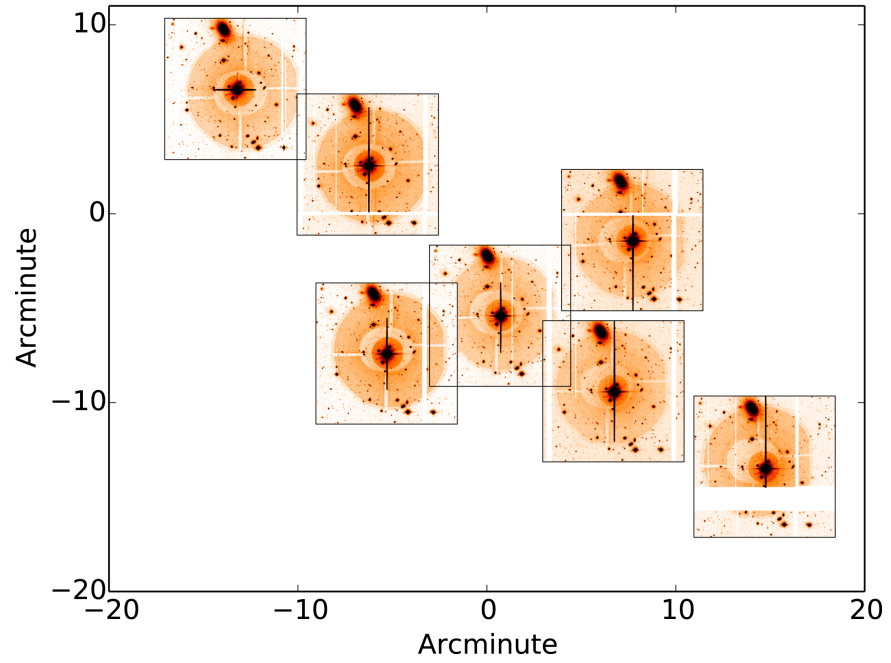

Fig. 2. Example of ghosts around a bright star close to the galaxy PGC 028887 (located to the north of the star). Each stamp corresponds to one of the 7 individual images obtained for that field and centered on the star. The positions of the stamps on the main panel reflect the observation strategy used for the MATLAS survey, making large offsets between the individual images. Note how the position of the main reflection annulus with respect to the star changes from one image to the other.

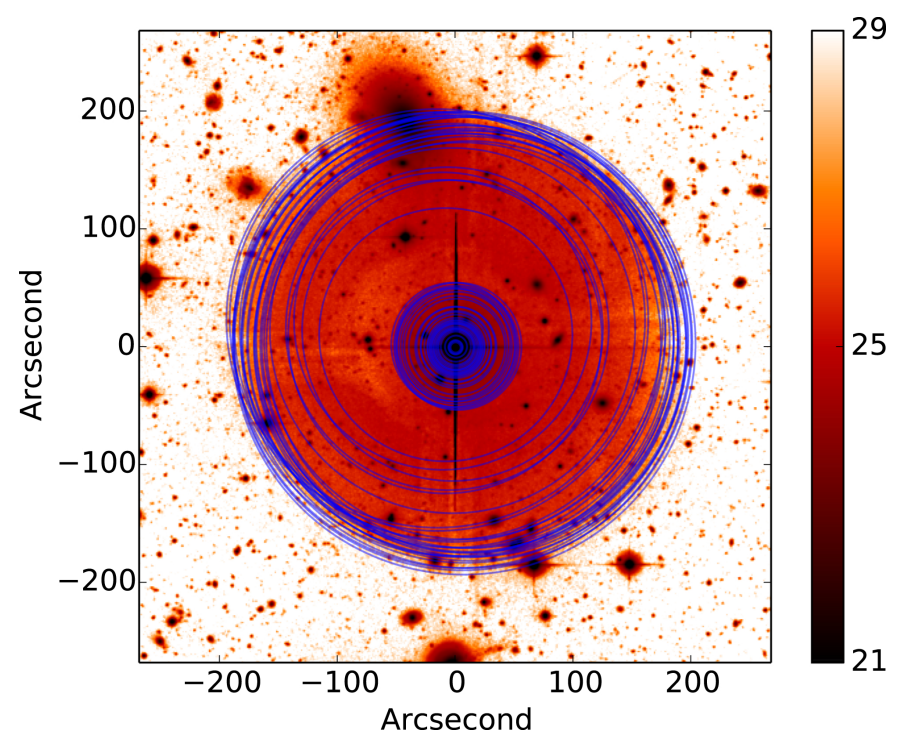

Fig. 3. Ghost halo of the star shown in Fig. 2, as seen in the stacked image. The blue circles correspond to the boundary of the individual disks used for the manual modeling of the halo.

regime, and without any AO facility, the shape of the inner PSF is, at first order, governed by the seeing of the atmosphere. Far out, its outer wings are shaped by the instrumental and, especially for MegaCam, by the multiple, thousands of internal reflections.

Building this type of PSF is not straightforward. A physical modeling of the PSF, taking into account the complex light path between the different optical elements of the camera, can be envisioned by ray tracing techniques, for instance. However, it would be computationally expensive to determine these PSF models for different locations on the image and for different wavelength ranges. Instead, empirical methods are used, and the PSF is directly derived from the science exposures. One way is to take numerous short exposure images, preventing saturation, 


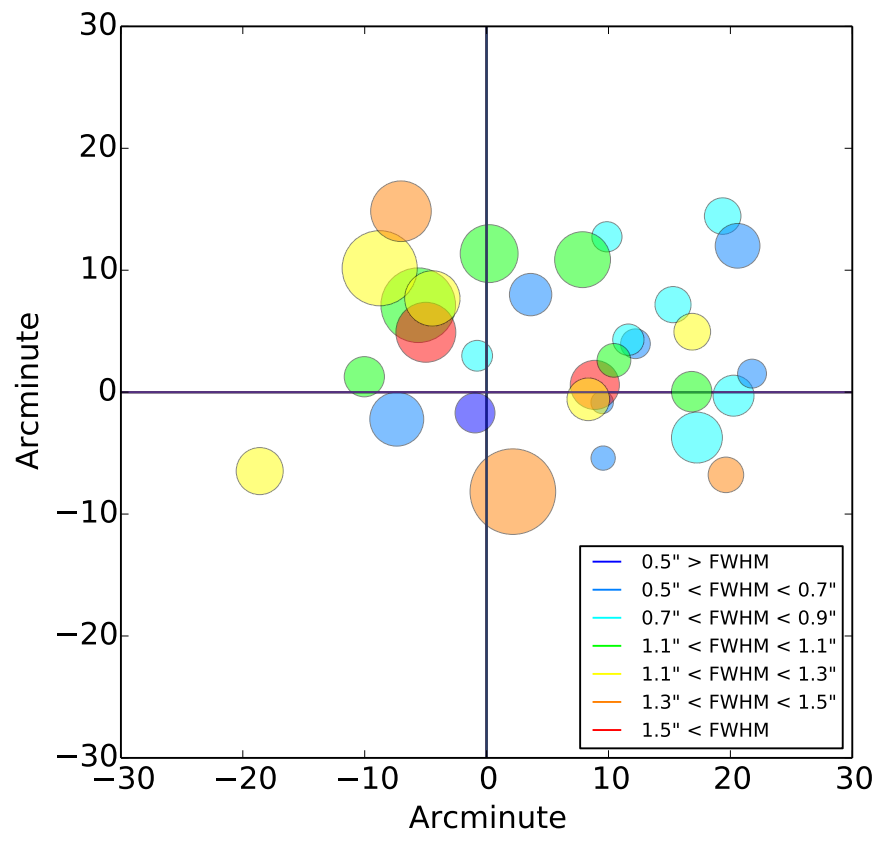

Fig. 4. Effect of the location of the reference star used for the $r$-band PSF modeling on the ghost strength. All stars used for the PSF modeling are plotted together. The disk position corresponds to the spatial offset of each star with respect to the center of the stacked images. Its size is proportional to the intensity of the star halo determined at a radius of $100^{\prime \prime}$, after a normalization of the profiles. Its color indicates the full width at half maximum (FWHM) of the star (with a color code given in the legend), to take into account the possible additional effect of the seeing.

and to stack them until the signal-to-noise $(\mathrm{S} / \mathrm{N})$ in the outer part of the PSF is high enough to model the wings (Janowiecki et al. 2010; Trujillo \& Fliri 2016). This type of calibration requires dedicated observations, matching the seeing conditions of the science observations, and thus a lot of telescope time. Alternatively, we can stack numerous non-saturated stars from one or, if necessary, several science exposures, but this process would smooth the non-symmetric features of the PSF. Indeed they vary with position and time.

We choose here a hybrid method. To create the PSF model, we build the inner profile by stacking many faint stars on the field and the outer profile by manually modeling the ghosts of a bright neighbor star; we then merge the inner and outer profiles. The inner parts of the PSF are generated using SExtractor (Bertin \& Arnouts 1996) and PSFex (Bertin 2011). Since the ellipticity, and FWHM of the PSF vary across the field of view, the reference stars were chosen within a box of $20^{\prime} \times 20^{\prime}$, centered on the target galaxy. This generally allows us to build the inner PSF model up to a radius of $\sim 6.5^{\prime \prime}$. The radius is reduced when the number of stars available for the PSF model with PSFex is insufficient and the $\mathrm{S} / \mathrm{N}$ is too low.

For the outer regions of the PSF, we directly exploit the depth of the MegaCam images, which enables us to model the light profile of bright (saturated) stars up to several arcmin. In most cases, the closest bright star to the target galaxy that exhibits prominent ghosts was used for the PSF-fitting procedure. The positions of the various selected stars are shown in Fig. 4. The wings of the PSF are computed with a similar manual method to the one used to remove the stellar ghosts (see Sect. 2.2), i.e. using a series of annuli with discrete intensity. One difference, however, is that the fitting procedure was continued to a smaller radius, i.e. $\sim 4.5^{\prime \prime}$.
With this method, the outer PSF is azimuthally averaged. Getting away from the center of the MegaCam field, the ghosts are less and less centered on the star. For this reason, in our star profile fitting procedure, the center of the annuli are allowed to be shifted. To build our PSF model, the annuli were, however, artificially re-centered. Indeed, the vast majority of our target galaxies are located close to the image center at a position where the ghost halo is centered on the star. With that approximation, we make sure that the deconvolution of the galaxy is carried out with a PSF with a shape that is close to the expected one.

Merging the inner and outer profiles of the PSF model requires a normalisation in the overlapping region. The normalisation factor is computed as the median ratio of the pixel values in a common region with an angular size of 1.1" (i.e. 6 pixels).

Figure 5 presents the typical profiles of the PSF models, for various seeing conditions and the two $g$ and $r$ bands. All PSF models were normalized by their estimated flux at 3 ", and combined within each seeing bin, and a standard deviation was then computed. The standard deviation curves shown in the figure indicate that the profiles are not uniform for a given seeing, band, and star magnitude. A typical error of $30 \%$ at radius of $100^{\prime \prime}$ is measured. This could first be due to uncertainties in the merging process of the inner and outer profiles of the PSFs, and to the spatial variations of the PSF wings. The impact of the latter effect is illustrated in Fig. 4 and further discussed in Sect. 4.1.

Though the intensity scaling used in our figures makes the ghost halos appear prominent, their contribution to the total flux is very limited: the flux integrated between $\sim 50-200^{\prime \prime}$ accounts for only $1.6 \%$; $98 \%$ of the flux is concentrated within the inner $10^{\prime \prime}$. We found that for a given seeing range, at a radius above $75^{\prime \prime}$, the $r$-band reflected light becomes more intense than in the $g$ band. This is mainly due to the CCDs being more reflective in the $r$ band. This wavelength dependence of the PSF creates artificial reddening of the outer profiles of galaxies.

More surprisingly, for a given band, the PSF models that correspond to worse seeing conditions have more prominent halos. The difference is significant: about $45 \%$ at $100^{\prime \prime}$, while the standard deviation per seeing bin is $\sim 30 \%$. We checked that a normalization of the flux with an aperture of $10^{\prime \prime}$, instead of $3^{\prime \prime}$, does not change the results.

Understanding the origin of these trends is beyond the scope of this study and will be discussed in a future paper that presents a physical, rather than empirical, modeling of the PSF. In any case, the strong wavelength, seeing, and position-dependence of the PSF wing intensity presented here further illustrates the need to have specific PSF models for each observation condition. However, as described in Sect. 2.2, obtaining a full PSF model with our manual empirical method is time-consuming. For that reason, instead of creating one PSF for each image of our large sample, we generated one PSF for each band and for different seeing conditions, ranging from $0.5^{\prime \prime}$ to $1.7^{\prime \prime} 3$. A database of 71 PSF models was thus generated.

\subsection{Image deconvolution}

To remove the ghost halos that are due to scattered light, we directly deconvolved our images using the very large kernels (PSFs) presented in Sect. 2.3. The deconvolution technique on large images with large kernels has two downsides: (1) it requires a large computation time even for low numbers of iterations (2) the deconvolved images exhibit irregularities, i.e. wiggles and

\footnotetext{
3 Note however that, for the vast majority of the MATLAS fields, the $r$-band seeing ranges between $0.5^{\prime \prime}$ and $1^{\prime \prime}$.
} 

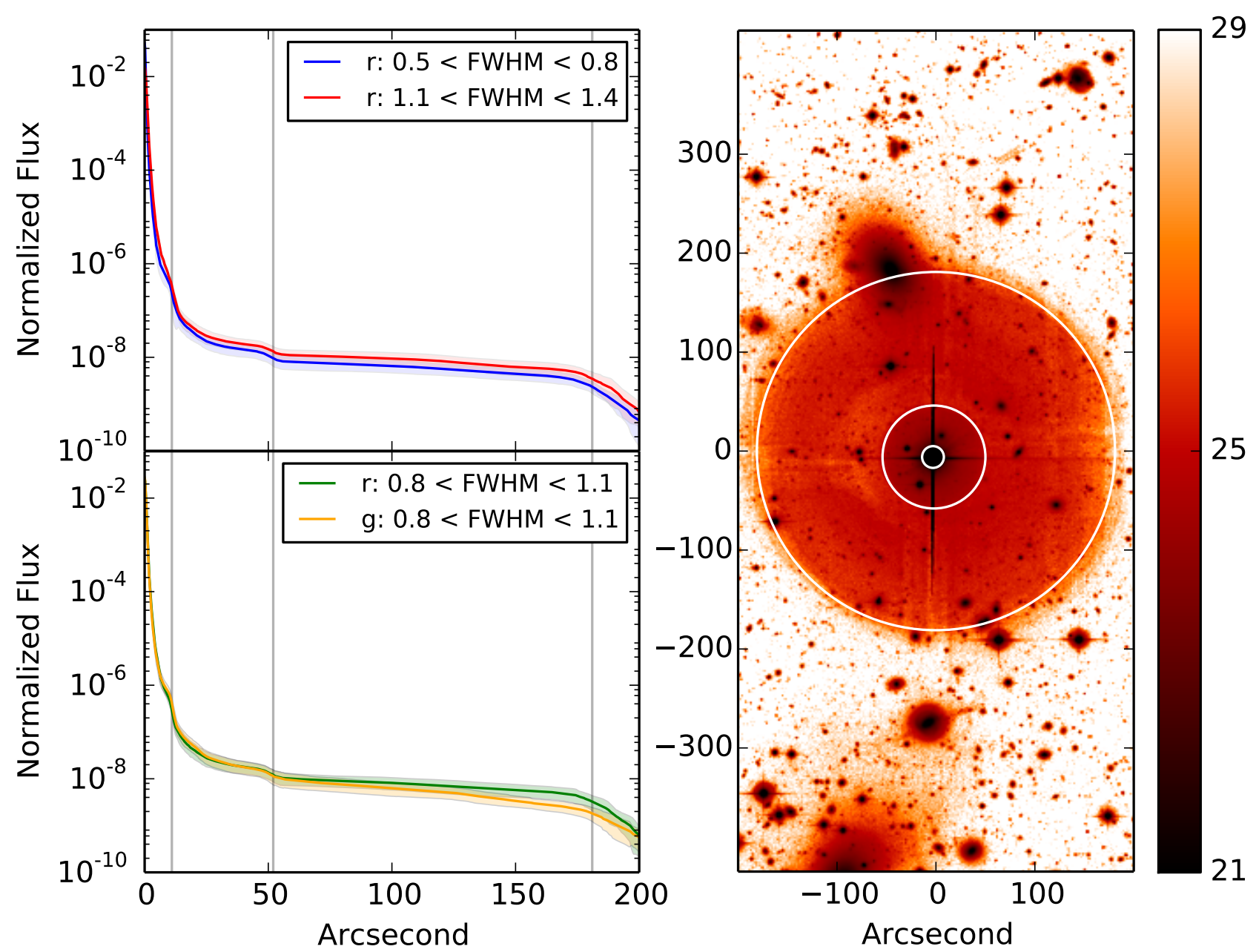

Fig. 5. Mean normalized profile of the $r$-band PSF model profiles for two bins of seeing (top-left); mean profile of the $g$-and $r$-band profiles for a specific seeing bin (bottom-left) and the $r$-band surface brightness map of a typical stellar ghost (right). The standard deviation of each profile is shaded. The surface brightness scale in $\operatorname{mag~arcsec}^{-2}$ is given to the right. The map is blurred with a Gaussian $\left(\operatorname{sigma}=0.55^{\prime \prime}\right)$ to enhance the faint regions. The vertical black lines in the plots to the left indicate the radii with sharp breaks in the profiles. Their positions on the surface brightness map are indicated by the white circles.

noise on small scales. This second issue is intrinsic to the technique and unavoidable since the PSF model can never be perfect. We have, however, dramatically reduced the computing time using specific python operators and solvers for high-performance computing.

The deconvolution method described here uses open source PyOperators, in particular convolution operators and equation solvers that were optimised to handle large matrix calculations. This allows the process to reach high iterations in a relatively short time. More specifically, the code uses a conjugate gradient method; the maximum iterations and the tolerance level for the residual are controlled by the user. We note that the cost of solving the minimization equations directly by a Cholesky decomposition would be prohibitive. The iterative preconditioned conjugate gradient method is more efficient.

The noise in the deconvolved images, which is due to the noise in the original images plus the imperfections in the representation of the PSF by the kernel, is regulated by a regularisation parameter. In addition, a normalisation parameter ${ }^{4}$ is given to help reach the solution with a low number of iterations.

\footnotetext{
4 Determined for each image as 1 divided by the square of the sigma of the sky background.
}

\subsection{Determining the galaxy profiles}

To derive the galaxy profiles, we used the following routine. First, the small objects (faint stars and background galaxies) were subtracted from the images, after having been identified by SExtractor and replaced by values of the surrounding background. Bad regions (including prominent CCD gaps, galactic cirrus, bright objects, etc.) were then manually masked. Initial photometric parameters were estimated by GALFIT, and the modeling of the galaxy itself was made by the ellipse task in IRAF. In the ellipse-fitting procedure, the position angle and ellipticity parameters were set free, except for galaxies with prominent bar-like structures, dust lanes, and bright clumps. The ellipse procedure usually breaks at locations where bright clumps within the galaxy or fine structures (tails, shells) become prominent. To force the determination of the profile farther out, a maximum semi-major axis was given. The profiles were however truncated at a fixed level of the measurement error, as described in Sect. 3.2.

The large scale sky background variations are the principle sources of systematic uncertainties in the light and color profile determination. Scattered light that is due to Galactic cirrus or clustered stars generate large fluctuations across the field. The determination of the average background around the target 
galaxy and its error is thus not straightforward. The code we developed for this process samples the background in 190 homogeneously distributed circular regions (150 pixel diameter) around the galaxy. The sky value and standard deviation is derived for each of these regions by IRAF's centroid sky fitting algorithm. Regions polluted by CCD gaps, stellar ghosts, or strong cirrus contamination are identified based on the value of the standard deviation and rejected in a two iteration process. For the remaining regions, the robust mean and sigma for the sky is calculated with the biweight_mean in IDL (Landsman 1993).

The local errors are directly given by the ellipse-fitting task. The error bars attached to the light and color profiles combine the systematic and local errors ${ }^{5}$ quadratically.

\section{Results}

In this section we present the deconvolution results on simulated images of galaxies and real images of NGC 3489, NGC 3599 , and NGC 4274. The analysis of the full sample will be presented in a future paper.

\subsection{Simulations}

The deconvolution technique was tested on convolved images of simulated galaxies. A single component galaxy model was created with GALFIT (Peng et al. 2002, 2010) and inserted onto the images, leaving enough padding to avoid any boundary effects in the convolution and deconvolution processes. The simulated galaxy has the following characteristics, typical of those of the early-type galaxies in our sample (see Table 1).

- Sérsic Index: 4.

- Integrated magnitude: 12.1 within a $5^{\prime \prime}$ aperture.

- Effective radius: 22.3".

- Ellipticity: 0.5.

The intrinsic simulated galaxy image was convolved by the convolution tool in PyOperators with a PSF that was obtained from a real $r$-band image. Its FWHM of $0.81^{\prime \prime}$ is close to the average seeing in $r$ band for the whole MATLAS survey. The deconvolution was performed on the convolved image with the code described in Sect. 2.4. No regularisation parameter was set since the simulated image is noiseless. The deconvolution was tested with different numbers of iterations, ranging between 25 to 20000 . Figure 6 shows that convergence in the outskirts of the galaxy is already reached with a small number of iterations $(\sim 100)$. As expected, recovering the true inner profile, however, requires a much higher number of iterations.

Figure 7 presents the projected images of the intrinsic, convolved, and deconvolved galaxy. It illustrates how light scattering not only change fluxes but also affects the outer isophotes of the galaxy: the halo gets artificially round shaped, especially between the 26th and 27th mag $\operatorname{arcsec}^{-2}$ isophotes. This effect can vary from galaxy to galaxy depending on the size, ellipticity, and brightness of the nucleus. As shown in the figure, the deconvolution technique is able to recover the intrinsic shape of the modelled galaxy.

\subsection{Observations: NGC 3489, NGC 3599, and NGC 4274}

The deconvolution technique was then applied to the images of real galaxies NGC 3489, NGC 3599, and NGC 4274, chosen for

\footnotetext{
5 Note that in the literature, the error on the sky background is usually neglected, resulting in much smaller error bars than those shown in this paper.
}

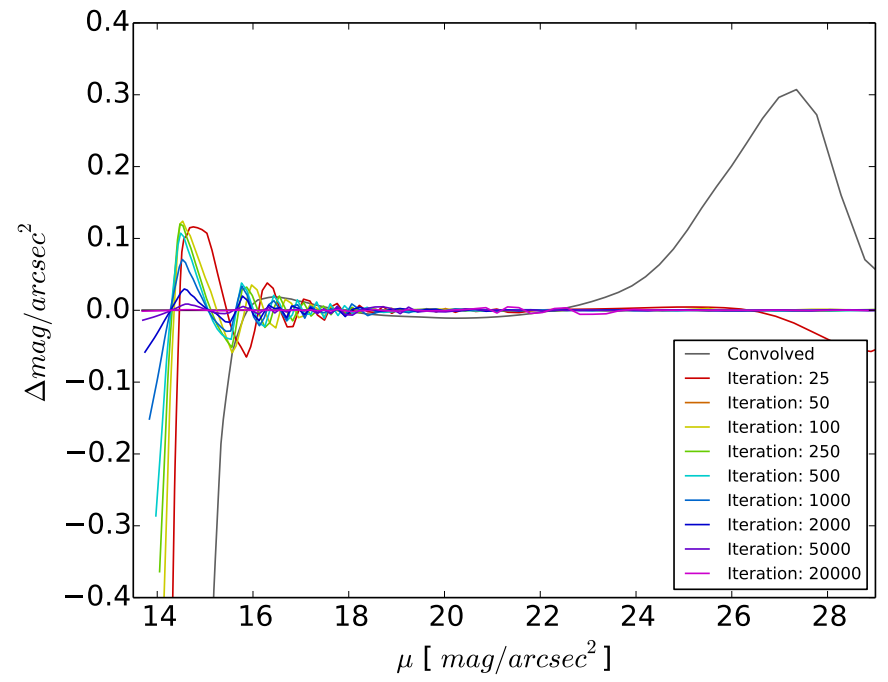

Fig. 6. Effect of the PSF on the profiles of simulated galaxies. The black curve shows the surface brightness difference between the convolved and original profiles of the simulated galaxy as a function of local surface brightness. The image is convolved with an $r$-band MegaCam PSF model and an FWHM equal to 0.81". The offset becomes significant above $25 \mathrm{mag} \operatorname{arcsec}^{-2}$ and reaches $0.3 \mathrm{mag} \operatorname{arcsec}^{-2}$ at the $27 \mathrm{mag} \operatorname{arcsec}^{-2}$ isophote. The colored curves show the difference between the deconvolved and original profiles for different numbers of iterations (given in the box).

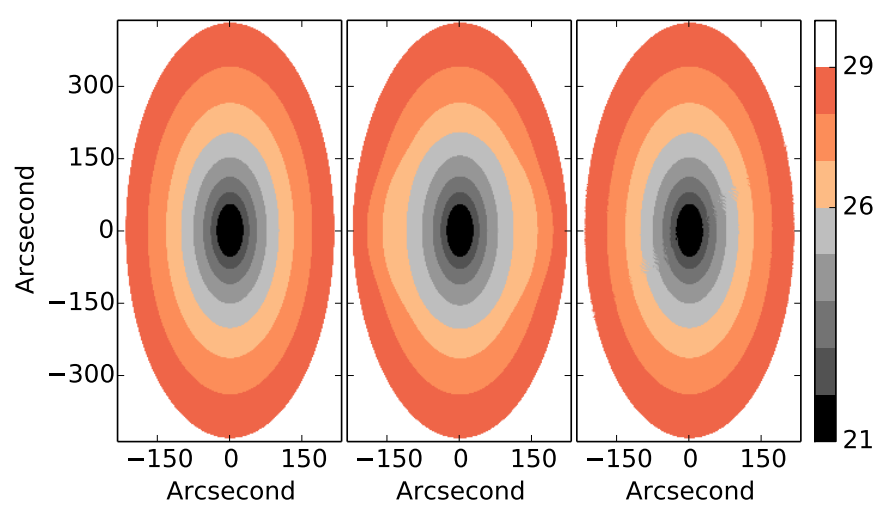

Fig. 7. Surface brightness maps of the original (left), convolved (middle), and deconvolved (right) images for the simulated galaxy. The color bar to the right represents the surface brightness level. The image is convolved with a PSF in $r$ band with $F W H M=0.81^{\prime \prime}$, and the deconvolved image is obtained after 20000 iteration steps by the solver.

being representative of the variety of objects in the MATLAS survey. Their properties are listed in Table 1.

NGC 3489 exhibits a prominent ghost that can be seen directly in the surface brightness and color maps as a roundish, reddish structure with a radius of 3.5 arcmin, typical of that surrounding bright stars (see Fig. 8).

We performed the deconvolution on the $g$ - and $r$-band images of NGC 3489, introducing a regularisation parameter this time. The technique successfully eliminates the artificial red halo after 500 iterations $^{6}$.

6 For real images, a higher number of iterations does not help to get rid of the wiggles generated by the deconvolution technique in the inner regions. Noise in the initial images and imperfections in the PSF modeling combine to prevent any efficient correction. For our purposes, the correction of the outer regions from light scattering, these artificial irregularities in the deconvolved image are not a problem. 

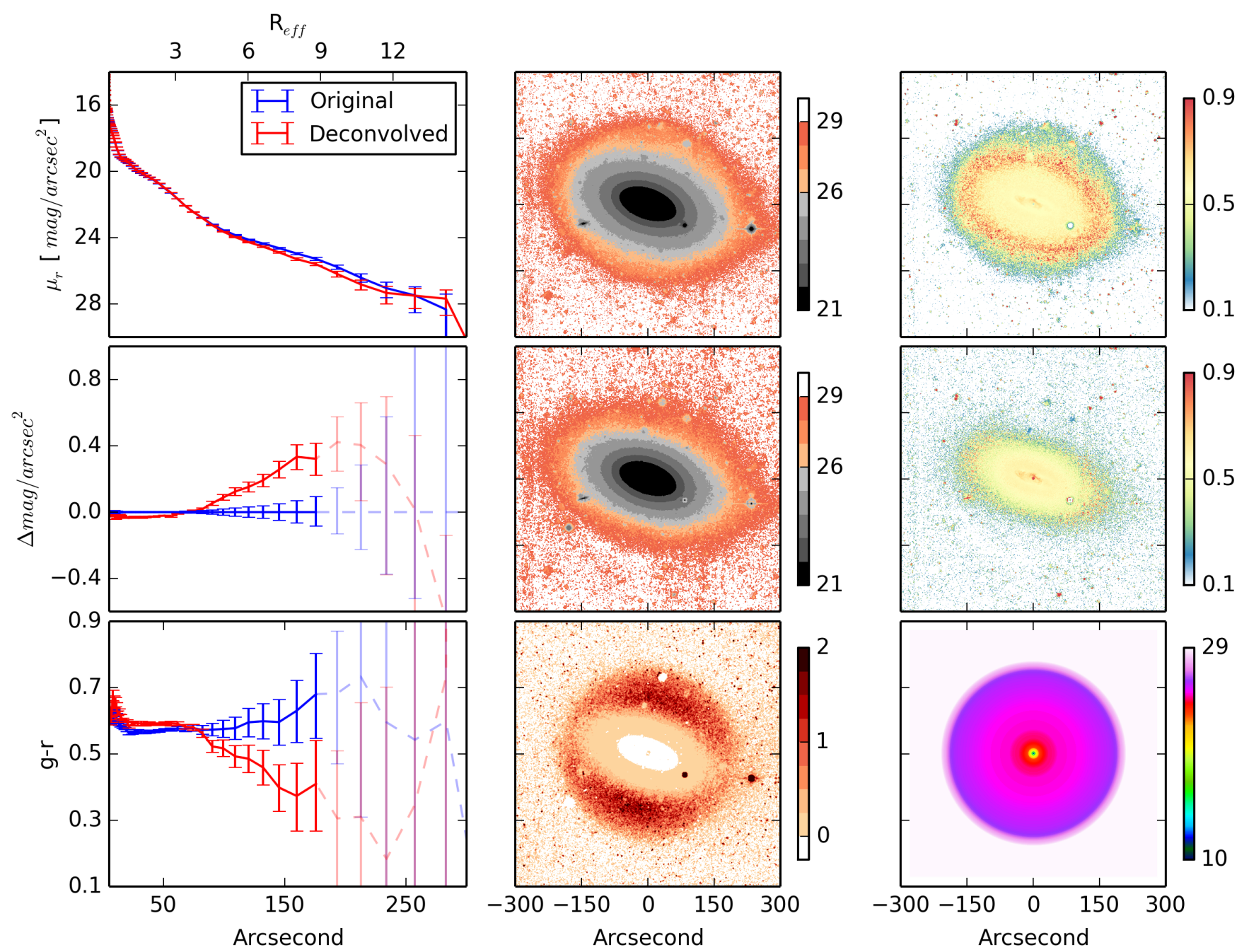

Fig. 8. Deconvolution technique applied to NGC 3489. Left column: original and deconvolved galaxy profiles in $r$ band (top), the difference between the profiles (middle) and original and deconvolved $g-r$ color profiles (bottom) as a function of semi-major axis. Middle column: original (top) and deconvolved (middle) surface brightness maps in $r$ band, and the difference between the maps (bottom). Right column: original (top) and deconvolved (middle) $g-r$ color maps and the $r$-band surface brightness map of the PSF (bottom) that was used for the deconvolution. All maps, except PSF, were smoothed with a Gaussian kernel of sigma $=0.55^{\prime \prime}$ to enhance the visibility of the faint regions.

Table 1. Properties of the tested galaxies.

\begin{tabular}{lccc}
\hline \hline & $R_{\text {Eff }}$ & Central brightness & Integrated magnitude \\
\hline NGC 3489 & 19.9 & 14.3 & 11.7 \\
NGC 3599 & 20.8 & 16.2 & 13.5 \\
NGC 4274 & 58.0 & 16.6 & 13.1 \\
\hline
\end{tabular}

Notes. Column (2): effective radius taken from the literature in arcseconds (Cappellari et al. 2013; Vika et al. 2013); Col. (3): central brightness of the light profile in $r$-band in mag $\operatorname{arcsec}^{-2}$; Col. (4): integrated magnitude within $5^{\prime \prime}$ (aperture) in $r$-band.

The profile in the $r$ band is corrected by up to $\sim 0.4 \mathrm{mag} \operatorname{arcsec}^{-2}$ at the $26 \mathrm{mag} \operatorname{arcsec}^{-2}$ isophote, corresponding to about $9 R_{\mathrm{Eff}}$. Because of the wavelength dependence of the PSF, the color profile is also affected by the light scattering. At the 26th mag arcsec ${ }^{-2}$ isophote, the deconvolution makes the $g-r$ color bluer by 0.2 mag. This results in an overall change in the color gradient: while the initial profile indicated an (artificial) reddening, the corrected one instead shows an increase in blueness. The change in the color profile becomes significant, i.e. larger than the error bar, beyond $5 R_{\mathrm{Eff}}$. The error bars of the color profiles are calculated by taking the square root of the sum of the square of each band's error.

NGC 3599 is representative of the lowest mass galaxies in the MATLAS/ATLAS ${ }^{3 \mathrm{D}}$ sample (see Table 1). Results for this galaxy are shown in Fig. 9. Both the light and the color profile show almost no change after deconvolution and the possible correction in the outermost part stays within the error bars owing to the low $\mathrm{S} / \mathrm{N}$ level in this region.

Finally the technique was tested on a late-type galaxy (LTG). LTGs that are not seen face-on have higher ellipticity and thus a shape that differs from the roundish ones that are characteristic of ghost halos. Scattered light is more likely to become dominant along the minor axis. LTGs are thus good cases to test the deconvolution technique. Although the MATLAS survey targets lenticular and elliptical galaxies, a number of spiral galaxies are present in the one square degree MegaCam field centered on the ETGs. At their location, often away from the center of the field, the PSF wings may be very different from the inner ones and, in particular, the ghost halos may no longer be concentric. 

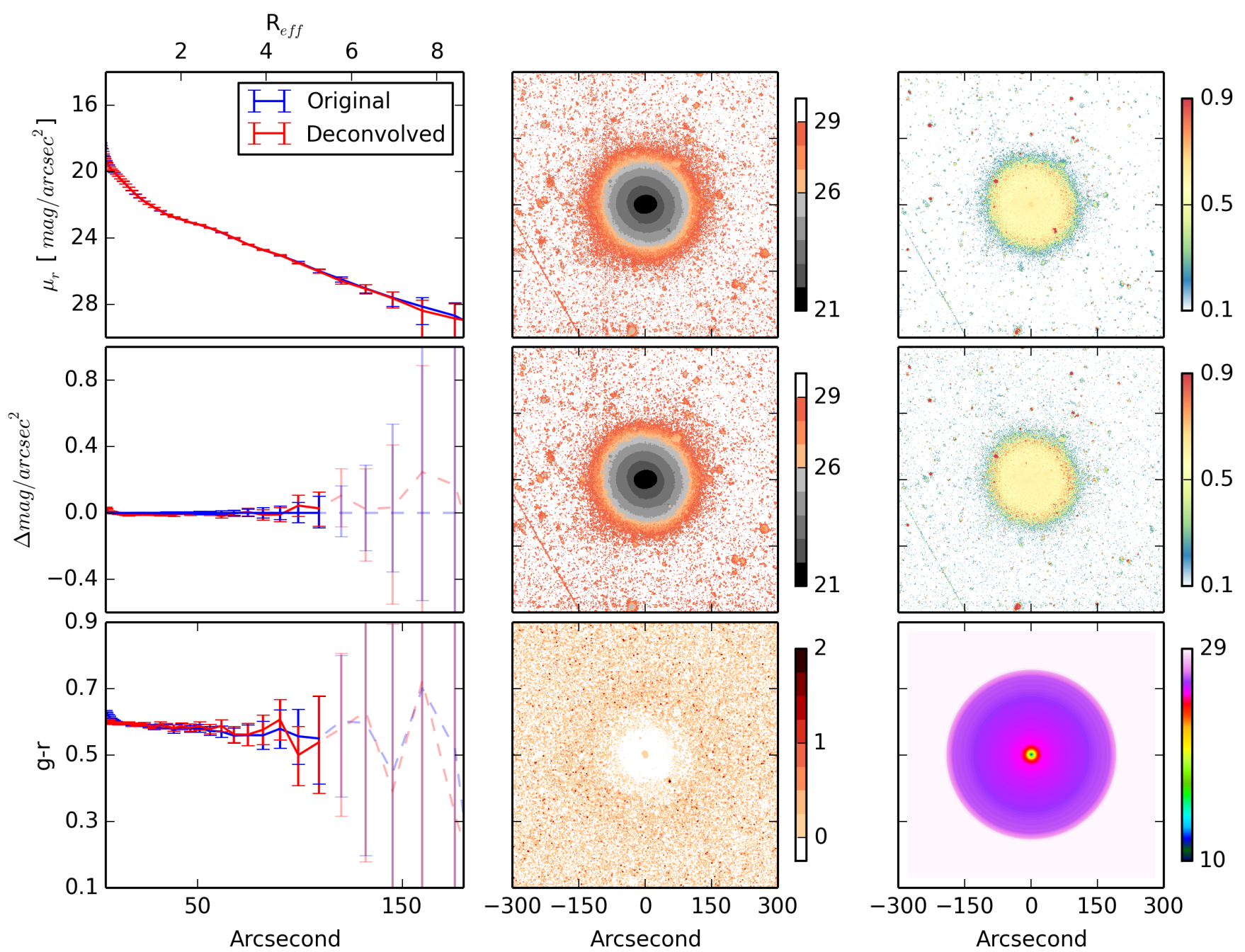

Fig. 9. Deconvolution technique applied to NGC 3599. See caption of Fig. 8 for details.

This is an opportunity to test the deconvolution method for this more complex configuration. Here, we present as a test case LTG NGC 4274, which is located in the field of ETG NGC 4278. An accurate PSF modeling was performed and, contrary to the previous cases, the centers of the halos were allowed to change positions. The results are shown in Fig. 10. The deconvolution removed most of the original prominent ghost around the galaxy (but owing to the position of the galaxy that is far from the center, not centered on it), but a faint residual is still visible, likely the result of the imperfect modeling of the PSF.

\section{Discussion}

In this section, we discuss the limitations of the deconvolution method, in particular owing to the imperfect PSF modeling and saturation. The overall importance of scattered light in the MATLAS survey is briefly addressed.

\subsection{Limitations of the method}

The ability to recover the original profile in simulated images, and the removal of the ghosts around galaxies in real images testify to the reliability of the deconvolution technique to achieve our primary goals: studying the light and color profiles of galaxies at large radius. However, as emphasised before, in all convolution/deconvolution techniques, having a perfect knowledge of the PSF is critical. Our modeling of the PSF that is based on stars located within the science frames has some limitations.

First, the outer profile of each PSF is derived from a single bright star in the image that is not located at the exact position of the galaxy; in most cases, it is selected as the closest bright star to have a prominent stellar ghost. The PSFs are subsequently built for the center of the images and the reflections are modeled as concentric halos. The latter hypothesis is justified at the position of the target galaxy, close to the center of the MegaCam field, where no important decentering is observed. As illustrated in Sect. 3.2 in the case of the off-centered galaxy NGC 4274, even when allowing a decentering for the PSF model, the ghost subtraction is not perfect.

Besides, the PSF was derived from stacked images and not from the individual ones. Our observing strategy requires us to implement large offsets between them, up to 14 arcmin. So, as illustrated in Fig. 2, the shape of the PSF and associated ghost halos differ from one image to another. Thus, in principle, a PSF model needs to be built for each image, and the deconvolution made before stacking using this specific PSF. This procedure would, however, greatly increase the interaction and computation time, which would make our largely manual method impractical for the complete sample. As a tradeoff, a proper 

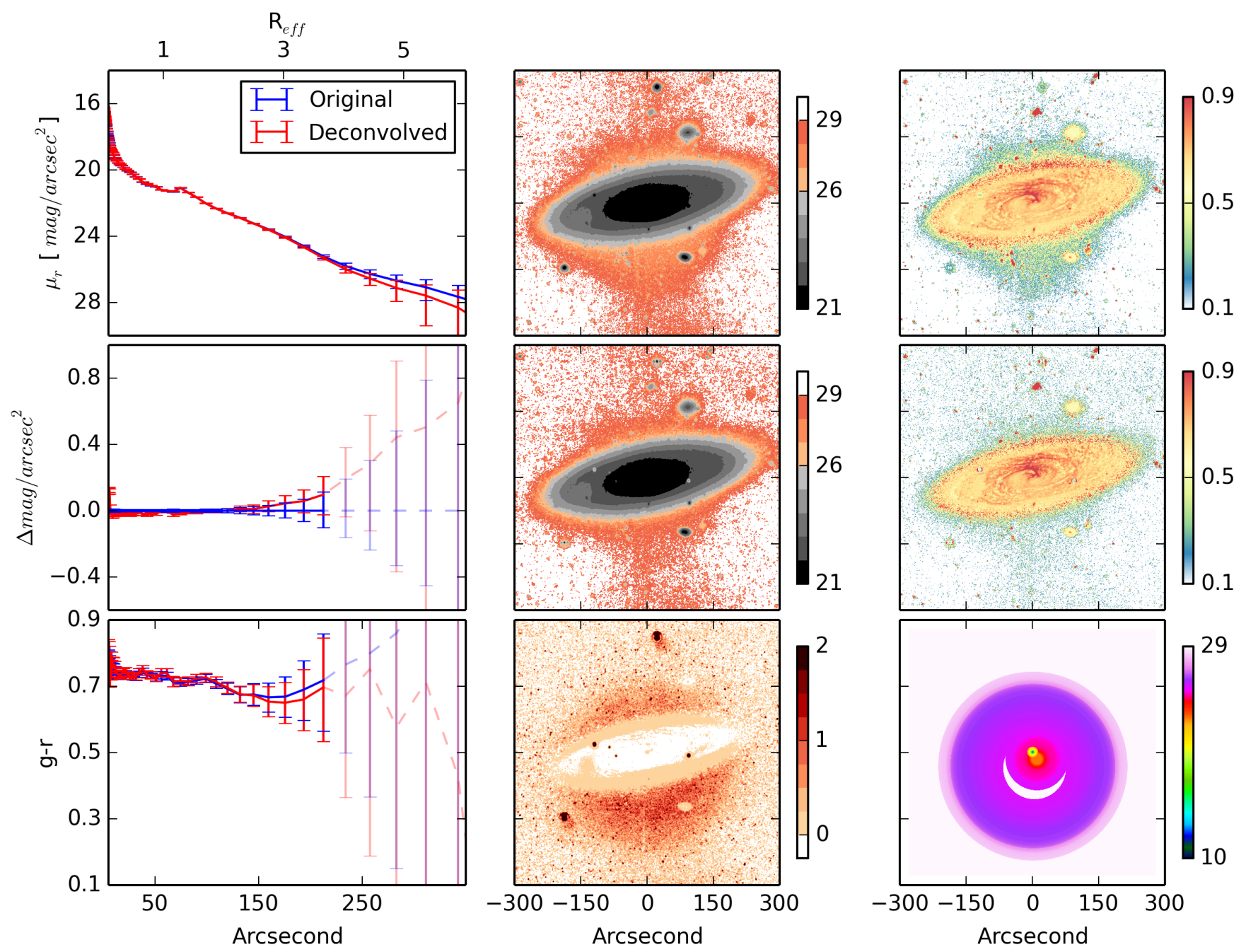

Fig. 10. Deconvolution technique applied to NGC 4274. See caption of Fig. 8 for details.

parametrisation of the ghost properties and determination of the parameter spatial variations using linear regressions could be done (Slater et al. 2009). An even better method would be to derive the PSFs with a physical model of the principle internal reflections in the camera. This would enable us to predict the PSF shape at each pixel. There are ongoing efforts to achieve this for the MegaCam camera.

We also note that, in practice, individual images most often lack signal to build the outer wings of the PSFs with the method described in Sect. 2.3 and, given, the low $\mathrm{S} / \mathrm{N}$ in the galaxy outskirts, the deconvolved image would be extremely noisy. The stacking procedure we used blurs the differences owing to the large offsets. However it does it the same way for the stars used to derive the PSF and the target.

\subsection{The importance of scattered light in the MATLAS survey}

The results of both simulations and observations show that the scattering should significantly vary from galaxy to galaxy. In the MATLAS sample, we expect to see a significant effect for some of the galaxies and almost none for others, depending on the brightness of the nucleus and also on the galaxy size. When stellar light and $\mathrm{S} / \mathrm{N}$ decrease, the correction for the scattered light becomes smaller than the error bars.
We created a simulated galaxy to test how the brightness of nucleus determines the amount of scattered light. The galaxies' properties are selected as described in Sect. 3.1. New galaxy models with higher central surface brightness are subsequently produced keeping the same light profile beyond $r=5^{\prime \prime}$, and steepening the inner profiles. All these images are then convolved with a real $r$-band PSF. The results are shown in Fig. 11. Brighter cores indeed create more artificial flux which, for the model tested here, starts to be significant, i.e. above $0.1 \mathrm{mag} \operatorname{arcsec}^{-2}$, beyond the $24 \mathrm{mag} \operatorname{arcsec}^{-2}$ isophote. The effect peaks at the $27 \mathrm{mag} \operatorname{arcsec}^{-2}$ isophote and decreases at lower surface brightness, i.e. at large radius. This is due to the fact that, for our model, the galaxy is more extended than the primary ghost (of 3.5 arcmin radius).

Conversely, if for whatever reason, including the presence of dust lanes, the nuclear light is dimmed, the ghosts become less prominent. Saturation is a special case for which the flux on the image is artificially dimmed, while the light of the nucleus arriving on the detector and level of internal reflections is unchanged. Here, the image should have a ghost contamination higher than anticipated from the measured central brightness. In other words, since the deconvolution process corrects the scattered light effect in the outer region based on the bright core, deconvolution of saturated sources leads to underestimated corrections. About $75 \%$ of the galaxies in the MATLAS long exposures are saturated and 

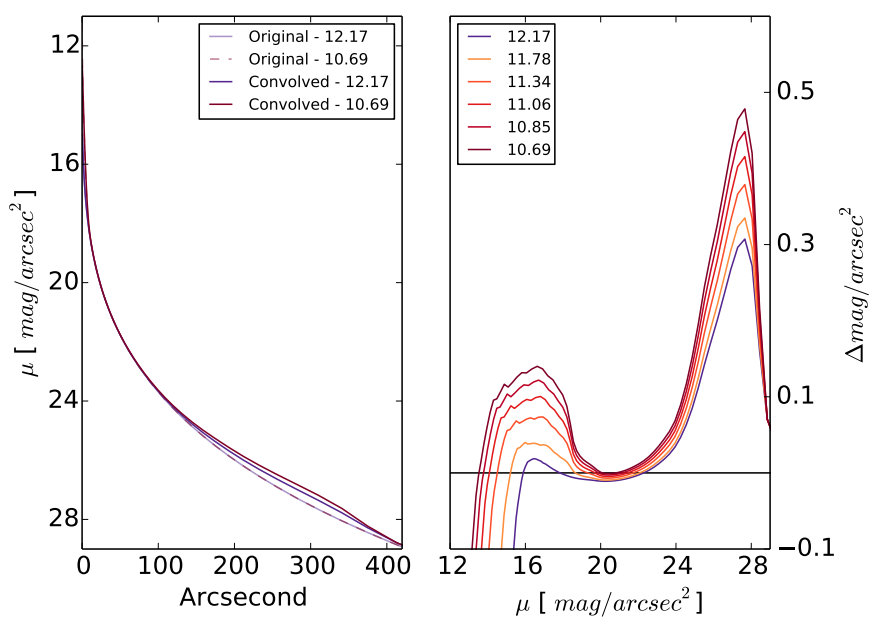

Fig. 11. Surface brightness profiles of the PSF-convolved and the original simulated galaxies (left) and difference between them as a function of surface brightness (right) for different central magnitude. Each line represents one of the simulated galaxies. The integrated magnitude within $5^{\prime \prime}$ (aperture) is written in the legend for each galaxy.

short exposures were acquired for all of them to recover the true inner profiles of the galaxies. The observations and the method of merging long and short exposure images to prevent saturation will be described in a future paper, together with the deconvolved light and color profiles of all MATLAS galaxies.

We note, however, that saturation does not necessarily affect the reconstructed light profile for all galaxies significantly. The effect is expected to be worse for relatively small, low luminosity galaxies with a bright saturated nucleus. These types of objects are actually rare in the ATLAS ${ }^{3 \mathrm{D}}$ sample.

\section{Conclusions}

In this paper, we have addressed the instrumental scattering effect in deep CFHT/MegaCam images. Our results are, however, relevant for the many cameras suffering from instrumental reflections (such as the one that equips the Sloan or ESO VST). These reflections produce wings (ghost halos) in the PSF, the importance of which depend on the wavelength and position in the field of view. With MegaCam, they are worse in the $r$ band. The wings of the PSF generate ghost halos around the galaxies, which may be directly visible on the images as round, reddish, disks. The scattered light affects the shape of the light and color profiles of galaxies. Our simulations show that the effect is worse between the 24 th and 28th mag $\operatorname{arcsec}^{-2}$ isophote, a region of prime importance since it accumulates tidal debris and thus traces the last accretion events of galaxies.

We have presented a method that is based on a direct deconvolution of the image and that is able to efficiently remove the ghost halos in a relatively low number of iterations. It requires a proper modeling of the PSF, which is made by combining the inner profile of stacked faint stars in the field and the outer profile of a bright (saturated) star close to the galaxy. We have built a PSF database that covers different seeing conditions, bands, and locations on the MegaCam field of view. With respect, the model-convolution technique also used to remove artificial halos in deep images, the deconvolution technique has the advantage of not requiring a proper, multi-component, modeling of the galaxy. It is thus much faster for large samples of complex galaxies, such as those studied as part of the MATLAS deep imaging survey. Intensive tests of the method have been made on simulations and on real galaxies presenting a variety of morphologies and sizes. Here, we have presented the results for three test cases: NGC 3489, NGC 3599, and NGC 4274.

In principle, the saturation in the cores of some of the galaxies averts the proper removal of the ghosts. A follow-up short exposure program has been completed to enable us to carry out a proper analysis. The full analysis of the sample will be presented in a future paper.

Acknowledgements. We thank the referee for his careful reading of the paper and for pointing out some issues that required further analysis. The paper is based on observations obtained with MegaPrime/MegaCam, a joint project of CFHT and CEA/DAPNIA, at the Canada-France-Hawaii Telescope (CFHT), which is operated by the National Research Council (NRC) of Canada, the Institute National des Sciences de l'Univers of the Centre National de la Recherche Scientifique of France, and the University of Hawaii. All observations were made as part of the service mode offered by the CFHT. We are grateful to the queue team for their dedication. We warmly thank C. Mihos, N. Regnault, C. Sandin, I. Trujillo, and L. Kelvin for the multiple discussions on ghost and scattered light effects. We are grateful to the CFHT staff for their dedication to the data acquisition.

\section{References}

Abraham, R. G., \& van Dokkum, P. G. 2014, PASP, 126, 55

Bertin, E. 2011, in Astronomical Data Analysis Software and Systems XX, eds. I. N. Evans, A. Accomazzi, D. J. Mink, \& A. H. Rots, ASP Conf. Ser., 442, 435

Bertin, E., \& Arnouts, S. 1996, A\&AS, 117, 393

Boulade, O., Charlot, X., Abbon, P., et al. 2003, in Instrument Design and Performance for Optical/Infrared Ground-based Telescopes, eds. M. Iye, \& A. F. M. Moorwood, Proc. SPIE, 4841, 72

Bullock, J. S., \& Johnston, K. V. 2005, ApJ, 635, 931

Cappellari, M., Emsellem, E., Krajnović, D., et al. 2011, MNRAS, 413, 813

Cappellari, M., Scott, N., Alatalo, K., et al. 2013, MNRAS, 432, 1709

Chanial, P., \& Barbey, N. 2012, in SF2A-2012: Proceedings of the Annual meeting of the French Society of Astronomy and Astrophysics, eds.

S. Boissier, P. de Laverny, N. Nardetto, R. Samadi, D. Valls-Gabaud, \& H. Wozniak, 513

Cooper, A. P., Cole, S., Frenk, C. S., et al. 2010, MNRAS, 406, 744

Duc, P.-A., Cuillandre, J.-C., Karabal, E., et al. 2015, MNRAS, 446, 120

Ferrarese, L., Côté, P., Cuillandre, J.-C., et al. 2012, ApJS, 200, 4

Iodice, E., Capaccioli, M., Grado, A., et al. 2016, ApJ, 820, 42

Janowiecki, S., Mihos, J. C., Harding, P., et al. 2010, ApJ, 715, 972

Landsman, W. B. 1993, in Astronomical Data Analysis Software and Systems II, eds. R. J. Hanisch, R. J. V. Brissenden, \& J. Barnes, ASP Conf. Ser., 52, 246 Martínez-Delgado, D., Gabany, R. J., Crawford, K., et al. 2010, AJ, 140, 962 Michard, R. 2002, A\&A, 384, 763

Mihos, J. C., Harding, P., Feldmeier, J., \& Morrison, H. 2005, ApJ, 631, L41 Miville-Deschenes, M.-A., Duc, P.-A., Marleau, F., et al. 2016, A\&A, 593, A4 Naab, T., Johansson, P. H., Ostriker, J. P., \& Efstathiou, G. 2007, ApJ, 658, 710 Oser, L., Ostriker, J. P., Naab, T., Johansson, P. H., \& Burkert, A. 2010, ApJ, 725,2312

Peng, C. Y., Ho, L. C., Impey, C. D., \& Rix, H.-W. 2002, AJ, 124, 266

Peng, C. Y., Ho, L. C., Impey, C. D., \& Rix, H.-W. 2010, AJ, 139, 2097

Pillepich, A., Madau, P., \& Mayer, L. 2015, ApJ, 799, 184

Sandin, C. 2014, A\&A, 567, A97

Sandin, C. 2015, A\&A, 577, A106

Slater, C. T., Harding, P., \& Mihos, J. C. 2009, PASP, 121, 1267

Trujillo, I., \& Fliri, J. 2016, ApJ, 823, 123

van Dokkum, P. G., Abraham, R., \& Merritt, A. 2014, ApJ, 782, L24

Vika, M., Bamford, S. P., Häußler, B., et al. 2013, MNRAS, 435, 623 